\title{
Dynamics of a three-dimensional charged particle dense bunch
}

\author{
H. Ye. Barminova* \\ Institute of Theoretical and Experimental Physics (ITEP), Moscow, Russia \\ A. S. Chikhachev \\ State Scientific Center "All-Russian Electrotechnical Institute” (VEI), Moscow, Russia \\ (Received 11 September 2012; published 28 May 2013)
}

\begin{abstract}
The behavior of a uniform charged particle bunch is studied. External and own bunch fields are taken into account. Two-dimensional and three-dimensional self-consistent problems are considered. The equations for bunch radii are obtained in the case of the bunch formed as a rotation ellipsoid. The model is proposed for the bunch with zero longitudinal emittance.
\end{abstract}

DOI: 10.1103/PhysRevSTAB.16.050402

PACS numbers: 41.85.Lc, 29.27.Bd

\section{INTRODUCTION}

For a lot of accelerator physics problems, the description of the charged particle bunch as an arbitrary ellipsoid is very useful. When accelerating, forming, and transporting the dense charged particle bunch changes its own shape under the influence of external and its own electromagnetic fields, so the relation between longitudinal and transverse dimensions of the bunch is changing widely. Thus, the problem of self-consistent ellipsoidal bunch description is of great interest [1]. Such a description allows one to predict the bunch behavior, in particular, to obtain the beam envelopes, without numerical simulation (therefore, more physically) or with numerical simulation when included as a model into the Poisson solver. Usually, for the bunch description the uniform density approximation is used [2], leading to the own fields linear dependent from coordinates. But in the three-dimensional case it is difficult to obtain exact nonstationary solutions for the kinetic distribution function if the bunch is considered as uniform charged ellipsoid $[1,3,4]$. In this paper the kinetic distribution functions are found which describe the bunch shaped as a rotation ellipsoid with uniform charge density for the various relations between transverse and longitudinal bunch sizes.

\section{2D PROBLEM}

Initially the development of nonstationary theory of the dense beams with strong own fields compared with the external ones was inspired by an exact solution of the twodimensional problem. Let us study the nonrelativistic motion of the bunch particles, and let us suppose that the coordinate system origin coincides with the bunch mass

\footnotetext{
*Elena.Barminova@itep.ru
}

Published by the American Physical Society under the terms of the Creative Commons Attribution 3.0 License. Further distribution of this work must maintain attribution to the author(s) and the published article's title, journal citation, and DOI. center. The particle motion in the central force field may be represented by the next equation:

$$
\ddot{r}=a(t) r+\frac{l^{2}}{r^{3}},
$$

where $l=M / m, M$ is an angular momentum, $r$ is a distance from symmetry axis. The function $a(t)$ characterizes the action of both the own bunch field and the external field in the case of the field forces linear dependent on transverse coordinates. The invariant that plays the Hamiltonian role may be written in the next form:

$$
I=[R(t) \dot{r}-\dot{R}(t) r]^{2}+\varepsilon_{0}^{2} \frac{r^{2}}{R^{2}}+l^{2} \frac{R^{2}}{r^{2}} .
$$

One can prove that $R(t)$ is the beam radius and $\varepsilon_{0}$ is the beam emittance. Taking into account the condition $\frac{d I}{d t} \equiv 0$ with Eq. (1) for $r$, we can obtain the equation for beam envelope (radius) $R(t)$ :

$$
\ddot{R}(t)=a(t) R+\frac{\varepsilon_{0}^{2}}{R^{3}} .
$$

The kinetic distribution function as a Vlasov's equation solution may be an arbitrary function of the motion integrals. In our case such integrals are $I$ and $l=M / m$. The beam density may by written as

$$
n=\int f(I, M) d p_{r} d p_{\varphi} .
$$

Taking into account that $p_{r}=m \dot{r}, p_{\varphi}=m r \dot{\varphi}=m \frac{l}{r}$, after conversation in the momentum space, one can obtain

$$
n=m^{2} \int \frac{d \operatorname{Idl} f(I, l)}{r R \sqrt{I-\frac{l^{2} R^{2}}{r^{2}}-\frac{\varepsilon_{0}^{2} r^{2}}{R^{2}}} .}
$$

The problem to find the distribution that gives the uniform beam density has not a single solution. We can write two expressions for function $f$, which lead to uniform density inside the beam (and, hence to linear dependence of the forces-the base for the invariant construction). In 
the first expression the function $f$ is not dependent on the angular momentum $l$ and should be considered as a "monochromatic" function:

$$
f=\frac{\kappa}{m^{2}} \delta\left(I-\varepsilon_{0}^{2}\right)
$$

The calculation of the integral over the phase space in which the radicand is positive leads to the next equality: $n=\frac{\pi \kappa}{R^{2}} \sigma\left(1-\frac{r^{2}}{R^{2}}\right)$, where $\sigma(x)$ is the Heaviside function: $\sigma(x)=1, x \geq 0, \sigma=0, x<0$. The parameter $\kappa$ is the normalization constant. One should note that this simple distribution is a particular case of Kapchinsky-Vladimirsky (KV) distribution [2]. In [5] another distribution was found leading to uniform density inside the beam too and in our notation it can be written as

$$
f=\frac{\kappa_{0}}{2 m^{2} \varepsilon_{0}} \frac{\sigma\left(\varepsilon_{0}^{2}-l^{2}\right)}{\sqrt{\varepsilon_{0}^{2}+l^{2}-I}} .
$$

Integration with this function leads to next expression for the beam density:

$$
n=\frac{\kappa_{0}}{R^{2}} \sigma\left(1-\frac{r^{2}}{R^{2}}\right)
$$

The difference between the distribution functions considered above affects on the calculation of the rms radial velocity variations (so, of the emittance). In the case of monochromatic distribution, we obtain

$$
\langle\dot{r}\rangle^{2}-\left\langle\dot{r}^{2}\right\rangle=\frac{\varepsilon_{0}^{2}}{2 R^{2}}\left(1-\frac{r^{2}}{R^{2}}\right)
$$

and in the case of distribution, presented in [5] we obtain

$$
\langle\dot{r}\rangle^{2}-\left\langle\dot{r}^{2}\right\rangle=\frac{2 \varepsilon_{0}^{2}}{3 R^{2}}\left(1-\frac{r^{2}}{R^{2}}\right) .
$$

In both cases the value $\varepsilon_{0}^{2}$ is a measure of the velocity spread (emittance), which vanishes at the boundary of the beam. One should note the more general character of the monochromatic distribution. It can be used for the description of the beam with elliptical cross-section. To satisfy this case, one should take the invariant as a $\operatorname{sum} I=I_{1}+I_{2}$, where

$$
\begin{aligned}
& I_{1}=\left(R_{x} \dot{x}-R_{x} x\right)^{2}+\varepsilon_{1}^{2} x^{2}, \\
& I_{2}=\left(R_{y} \dot{y}-R_{y}^{\dot{y}} y\right)^{2}+\varepsilon_{2}^{2} y^{2} .
\end{aligned}
$$

$R_{x}, R_{y}$ are the values of semiaxes of the elliptical crosssection. From the condition of the invariant conservation, one can obtain the equations for the semiaxes (radii). The presence of the $\delta$-function in the expression for the invariant leads to uniform density in the beam cross-section. Such a function and such a method were used in [6] for the channel with quadrupole systems.

\section{SPHERICAL BUNCH}

Let us consider a bunch with a spherical symmetry, coordinate system connecting with the bunch center. For simplicity, let us consider that there are no external fields, and the bunch mass center is not moving. In spherical coordinates the charged particle motion equations may be written

$$
\begin{gathered}
m r^{2} \dot{\phi} \sin ^{2} \theta \equiv \text { const }=M_{\varphi}, \\
m^{2}\left(r^{2} \dot{\theta}\right)^{2}+\frac{M_{\varphi}^{2}}{\sin ^{2} \theta} \equiv L>0, \\
\ddot{r}-a(t) r=\frac{L}{m^{2} r^{3}} .
\end{gathered}
$$

Here $M_{\varphi}, L$ are the motion integrals that follow from the given bunch symmetry, $M_{\varphi}$ is the projection of the whole momentum on the axis $Z, L$ is the total momentum in the second power, and $m$ is the mass of the particle. Equation (3) is valid only in the case of a sphere with uniform charge density, otherwise value $a$ depends not only on $t$ but on $r$. Using the relation $\operatorname{div} \vec{E}=4 \pi e n$, where $e$ is the electron charge and $n$ is the particle density, one can obtain for $a(t)$,

$$
a(t)=e^{2} N / m R^{3}(t),
$$

where $N$ is the total number of particles in the bunch, and $R(t)$ is the time-dependent bunch radius. For a complete description of the bunch it is necessary to find three integrals of the motion. In our case, the third integral may be found from Eq. (3), which describes the radial motion of the particle. Let us introduce an invariant which is similar to the invariant (2):

$$
I=[R(t) \dot{r}-R \dot{(}(t) r]^{2}+\lambda \frac{R^{2}}{r^{2}}+\varepsilon_{0}^{2} \frac{r^{2}}{R^{2}}
$$

$\left(\lambda=L / m^{2}\right)$. It is easy to obtain that $\frac{d I}{d t} \equiv 0$, if

$$
\ddot{R}-a(t) R=\frac{\varepsilon_{0}^{2}}{R^{3}(t)} .
$$

The distribution function for our bunch may be written as a function of the motion integrals- $\Phi\left(I, L, M_{\varphi}\right)$. Then during calculation of the particle density $n=$ $\int \Phi d p_{r} d p_{\theta} d p_{\varphi}$, let us replace integration variables by $I, \lambda=L / m^{2}, M_{\varphi}$. Here

$$
\begin{aligned}
& p_{\varphi}=m r \sin \theta \dot{\varphi}=\frac{M_{\varphi}}{r \sin \theta} \\
& p_{\theta}=m \dot{\theta}=\frac{1}{r} \sqrt{L-\frac{M_{\varphi}^{2}}{\sin ^{2} \theta}} \\
& p_{r}=m \dot{r}=\frac{m}{R} \sqrt{I-\frac{L}{m^{2}} \frac{R^{2}}{r^{2}}-\varepsilon_{0}^{2} \frac{r^{2}}{R^{2}}}
\end{aligned}
$$


So for the density we obtain

$$
\begin{aligned}
n= & \frac{m^{2}}{r^{2} R} \int_{\varepsilon_{0}^{2}\left(r^{2} / R^{2}\right)}^{\infty} d I \int_{0}^{\left(r^{2} / R^{2}\right)\left[I-\varepsilon_{0}^{2}\left(r^{2} / R^{2}\right)\right]} \frac{d \lambda}{\sqrt{I-\lambda \frac{R^{2}}{r^{2}}-\varepsilon_{0}^{2} \frac{r^{2}}{R^{2}}}} \\
& \times \int_{-\sqrt{\lambda} m \sin \theta}^{\sqrt{\lambda} m \sin \theta} \frac{\Phi\left(I, \lambda, M_{\varphi}\right) d M_{\varphi}}{\sqrt{\lambda \sin ^{2} \theta-\frac{M_{\varphi}^{2}}{m^{2}}}} .
\end{aligned}
$$

The integration limits in (12) are determined according to the requirement for expressions under the integral signs to be more or equal to zero. Let us find solutions that correspond to the case of the function $\Phi$ which is not dependent on $M_{\varphi}$. In this case the expression for density $n$ looks as

$$
\begin{aligned}
n= & \frac{\pi m^{3}}{r^{2} R} \int_{\varepsilon_{0}^{2}\left(r^{2} / R^{2}\right)}^{\infty} d I \\
& \times \int_{0}^{\left(r^{2} / R^{2}\right)\left[I-\varepsilon_{0}^{2}\left(r^{2} / R^{2}\right)\right]} \frac{d \lambda \Phi(I, \lambda)}{\sqrt{\frac{r^{2}}{R^{2}}\left(I-\varepsilon_{0}^{2} \frac{r^{2}}{R^{2}}\right)-\lambda}} .
\end{aligned}
$$

It should be noted that if we take $\Phi$ as $\Phi=\Phi_{1}(I) \Phi_{2}(\lambda)$, it leads to physically unreal result-negative density. First found in [7] (see also [3]), the solution for the function describing real physical conditions looks as

$$
\Phi(I, \lambda)=\frac{3 N}{4 \pi^{2} m^{3} \varepsilon_{0}^{2}} \frac{\sigma\left(1-I / \varepsilon_{0}^{2}+\lambda / \varepsilon_{0}^{2}\right)}{\sqrt{\left(1-I / \varepsilon_{0}^{2}+\lambda / \varepsilon_{0}^{2}\right)}} \sigma(\lambda) \sigma\left(\varepsilon_{0}^{2}-\lambda\right) .
$$

According to (13) and (14), one can obtain for the density

$$
\begin{aligned}
n= & \frac{\pi m^{3}}{r^{2} R} \frac{3 N}{\pi^{2} m^{3} \epsilon_{0}^{2}} \int_{0}^{\varepsilon_{0}^{2}} d \lambda \\
& \times \int_{\lambda\left(R^{2} / r^{2}\right)+\varepsilon_{0}^{2}\left(r^{2} / R^{2}\right)}^{\lambda+\varepsilon^{2}} \frac{d I}{\sqrt{I-\lambda \frac{R^{2}}{r^{2}}-\varepsilon_{0}^{2} \frac{r^{2}}{R^{2}}} \sqrt{\varepsilon_{0}^{2}+\lambda-I}} .
\end{aligned}
$$

In (15) the upper integration limit must be more than the lower limit, i.e., this integral is proportional,

$$
\begin{aligned}
\sigma\left(\lambda+\epsilon_{0}^{2}-\lambda \frac{R^{2}}{r^{2}}-\varepsilon_{0}^{2} \frac{r^{2}}{R^{2}}\right)= & \sigma(R-r) \sigma\left(\varepsilon_{0}^{2} \frac{r^{2}}{R^{2}}-\lambda\right) \\
& +\sigma(r-R) \sigma\left(\lambda \frac{R^{2}}{r^{2}}-\varepsilon_{0}^{2}\right) .
\end{aligned}
$$

Here the second summand in the right part is not essential, because the condition $\lambda \frac{R^{2}}{r^{2}}>\varepsilon_{0}^{2}$ (when $r>R$ ) leads to $\lambda>\varepsilon_{0}^{2}$. Finally, for the density we obtain

$$
n=\frac{3 N \pi m^{3}}{4 r^{2} R \pi^{2} \varepsilon_{0}^{2} m^{3}} \frac{\varepsilon_{0}^{2} r^{2}}{R^{2}} \sigma(R-r)=\frac{3 N}{4 \pi R^{3}(t)} \sigma[R(t)-r] .
$$

If the invariant depends on $M_{\varphi}$ as $\delta\left(M_{\varphi}\right)$, then the beam density cannot be uniform because the factor $\frac{1}{\sin \theta}$ appears.
So the density has a singularity when $\theta \rightarrow 0$ or $\theta \rightarrow \pi$. In this case the own forces will not have linear character, and there is incorrectness in [5]. In [5] the absence of the factor mentioned above means the calculations were made with some other dependence on the angular momentum, not with the $\delta$-function.

\section{ELLIPSOIDAL BUNCH}

A spherical model is unsuitable for description of a real bunch behavior in external fields due to spherical symmetry absence. So a bunch model should be built in the case of axial symmetry. The motion equations in cylindrical coordinates may be written as

$$
\ddot{r}+a_{\perp}(t) r=\frac{M_{\varphi}^{2}}{m r^{3}}, \quad \ddot{\zeta}+a_{\|}(t) \zeta=0 .
$$

Here $M_{\varphi}=r p_{\phi}=m r^{2} \dot{\varphi}$ is the motion equation integral, $r$ is the distance between the particle position and the axis, and $\zeta=z-z_{0}$ is the projection of the distance between the particle and bunch center on the axis $z$. Equations (18) are valid for the uniform density bunch. They permit to use two motion integrals:

$I_{1}=\left[R_{\perp}(t) \dot{r}-R_{\perp} \dot{(}(t) r\right]^{2}+\mu^{2} \frac{R_{\perp}^{2}}{r^{2}}+\varepsilon_{\perp}^{2} \frac{r^{2}}{R_{\perp}^{2}}$,

$I_{2}=\left[R_{\|}(t) \dot{\zeta}-R_{\|}(t) \zeta\right]^{2}+\varepsilon_{\|}^{2} \frac{\zeta^{2}}{R_{\|}^{2}}$.

Here $R_{\perp}(t), R_{\|}(t)$ are the transverse and longitudinal sizes of the bunch respectively, $\varepsilon_{\perp}, \varepsilon_{\|}$are the transverse and longitudinal emittances respectively, and $\mu=\frac{M_{\varphi}}{m}$. Invariants $I_{1}, I_{2}$ are constant, if $R_{\perp} R_{\|}$satisfy the equations

$$
\ddot{R_{\perp}}+a_{\perp} R_{\perp}=\frac{\varepsilon_{\perp}^{2}}{R_{\perp^{3}}}, \quad \ddot{R}_{\|}+a_{\|} R_{\|}=\frac{\varepsilon_{\perp}^{2}}{R_{\|^{3}}} .
$$

The density $n=\int f d p_{z} d p_{r} d p_{\varphi}$ may be represented by means of the motion integrals as follows:

$$
\begin{aligned}
n= & m^{3} \int \Psi\left(I_{1}, I_{2}, \mu\right) \frac{d \mu}{r} \frac{d I_{2}}{R_{\|} \sqrt{I_{2}-\varepsilon_{\|}^{2} \frac{\zeta^{2}}{R_{\|}^{2}}}} \\
& \times \frac{d I_{2}}{R_{\perp} \sqrt{I_{1}-\mu^{2} \frac{R_{\perp}^{2}}{r^{2}}-\varepsilon_{\perp}^{2} \frac{r^{2}}{R_{\perp}^{2}}}}
\end{aligned}
$$

(where $p_{z}=m \dot{z}, p_{r}=m \dot{r}, p_{\varphi}=m r \dot{\varphi}=M_{\varphi} / r$ ). Function $\Psi \geq 0$ must satisfy relation $n=n_{0} \sigma\left(1-\frac{r^{2}}{R_{\perp}^{2}}-\frac{\zeta^{2}}{R_{\|}^{2}}\right)$, e.g., it must result in uniform density inside the bunch and to zero density outside the bunch. The limits of integration should be determined from the requirement of positive values of the radicands in (21). Let us consider the next expression for distribution function $\Psi$ : 


$$
\begin{aligned}
\Psi\left(I_{1}, I_{2}, \mu\right)= & \frac{3 N}{2 \pi^{3} m^{3}} \frac{\varepsilon_{\perp}^{2}\left(1-I_{2} / \varepsilon_{\|}^{2}\right)^{2}}{\varepsilon_{\|}} \\
& \times \frac{\sigma\left(1-I_{2} / \varepsilon_{\|}^{2}\right) \sigma\left[\varepsilon_{\perp}^{2}\left(1-I_{2} / \varepsilon_{\|}^{2}\right)^{2}-\mu^{2}\right]}{\left[\varepsilon_{\perp}^{2}\left(1-I_{2} / \varepsilon_{\|}^{2}\right)^{2}-\mu^{2}\right]^{3 / 2}} \\
& \times \frac{\sigma\left[\varepsilon_{\perp}^{2}\left(1-I_{2} / \varepsilon_{\|}^{2}\right)+\mu^{2} /\left(1-I_{2} / \varepsilon_{\|}^{2}\right)-I_{1}\right]}{\sqrt{\left[\varepsilon_{\perp}^{2}\left(1-I_{2} / \varepsilon_{\|}^{2}\right)+\mu^{2} /\left(1-I_{2} / \varepsilon_{\|}^{2}\right)-I_{1}\right]}} .
\end{aligned}
$$

Integrating (21) with respect to $I_{1}$ and taking into account (22), we obtain

$$
\begin{aligned}
& \int_{\mu^{2}\left(R_{\perp}^{2} / r^{2}\right)+\varepsilon_{\perp}^{2}\left(r^{2} / R_{\perp}^{2}\right)}^{\varepsilon^{2}\left(1-I_{2} / \varepsilon_{1}^{2}\right)+\mu^{2} /\left(1-I_{2} / \varepsilon_{\|}^{2}\right)} \frac{d I_{1}}{\sqrt{I_{1}-\mu^{2} \frac{R_{\perp}^{2}}{r^{2}}-\varepsilon_{\perp}^{2} \frac{r^{2}}{R_{\perp}^{2}}}} \\
& \times \frac{1}{\sqrt{\varepsilon_{\perp}^{2}\left(1-I_{2} / \varepsilon_{\|}^{2}\right)+\mu^{2} /\left(1-I_{2} / \varepsilon_{\|}^{2}\right)-I_{1}}} \\
& =\pi \sigma\left(\varepsilon_{\perp}^{2}\left(1-I_{2} / \varepsilon_{\|}^{2}\right)+\frac{\mu^{2}}{1-I_{2} / \varepsilon_{\|}^{2}}-\mu^{2} \frac{R_{\perp}^{2}}{r^{2}}-\varepsilon_{\perp}^{2} \frac{r^{2}}{R_{\perp}^{2}}\right) \text {. }
\end{aligned}
$$

The last expression may be transformed as

$$
\begin{aligned}
& \sigma\left(\varepsilon_{\perp}^{2}\left(1-I_{2} / \varepsilon_{\|}^{2}\right)+\frac{\mu^{2}}{1-I_{2} / \varepsilon_{\|}^{2}}-\mu^{2} \frac{R_{\perp}^{2}}{r^{2}}-\varepsilon_{\perp}^{2} \frac{r^{2}}{R_{\perp}^{2}}\right) \\
& =\sigma\left(1-\frac{I_{2}}{\varepsilon_{\|}^{2}} \frac{\mu^{2}}{\varepsilon_{\perp}^{2}} \frac{R_{\perp}^{2}}{r^{2}}\right) \sigma\left(1-\frac{I_{I}}{\varepsilon_{\|}^{2}} \frac{r^{2}}{R_{\perp}^{2}}\right) \\
& \quad+\sigma\left(-1+\frac{I_{2}}{\varepsilon_{\|}^{2}}+\frac{\mu^{2}}{\varepsilon_{\perp}^{2}} \frac{r^{2}}{R_{\perp}^{2}}\right) \sigma\left(-1+\frac{I_{2}}{\varepsilon_{\|}^{2}}+\frac{r^{2}}{R_{\perp}^{2}}\right) .
\end{aligned}
$$

Here the condition $1>I_{2} / \varepsilon_{\|}^{2}$ was taken into account. The last summand in the right part is not essential because $1<\frac{I_{2}}{\varepsilon_{\|}^{2}}+\frac{r^{2}}{R_{\perp}^{2}}$ contradicts relations $\frac{I_{2}}{\varepsilon_{\|}^{2}}+\frac{\mu^{2}}{\varepsilon_{\perp^{2}}} \frac{R_{\perp}^{2}}{r^{2}}>1$ and $\varepsilon_{\perp}^{2}\left(1-\frac{I_{2}}{\varepsilon_{\|}^{2}}\right)^{2}>\mu^{2}$.

Let us do integration with respect to $\mu$. So

$$
\begin{gathered}
\int_{-\varepsilon_{\perp}\left(r / R_{\perp}\right) \sqrt{1-\left(I_{2} / \varepsilon_{\|}^{2}\right)}}^{\varepsilon_{\perp}\left(r / R_{\perp}\right) \sqrt{1-\left(I_{2} / \varepsilon_{\|}^{2}\right)}} \frac{d \mu}{\left[\varepsilon_{\perp}^{2}\left(1-\frac{I_{2}}{\varepsilon_{\|}^{2}}\right)^{2}-\mu^{2}\right]^{3 / 2}} \\
=\frac{2 r / R_{\perp}}{\sqrt{1-\frac{I_{2}}{\varepsilon_{\|}^{2}}-\frac{r^{2}}{R_{\perp}^{2}}}} \frac{1}{\varepsilon_{\perp}^{2}\left(1-\frac{I_{2}}{\varepsilon_{\|}^{2}}\right)^{2}} .
\end{gathered}
$$

Finally for the density, one can obtain

$$
n=\frac{3 N}{4 \pi R_{\perp}^{2} R_{\|}} \sigma\left(1-\frac{r^{2}}{R_{\perp}^{2}}-\frac{\zeta^{2}}{R_{\|}^{2}}\right) .
$$

First the distribution (22) was found in [7].

\section{SEMIAXIS EQUATIONS}

The potential of the ellipsoid with uniform charge density in vacuum is performed by the next expression [8]:

$$
\Phi=\frac{3 e N}{4} \int_{0}^{\infty} \frac{d y}{T(y)}\left(1-\frac{z^{2}}{R_{\|}^{2}+y}-\frac{r^{2}}{R_{\perp}^{2}+y}\right),
$$

where

$$
T(y)=\left(R_{\perp}^{2}+y\right) \sqrt{R_{\perp}^{2}+y} .
$$

From (24), one can obtain the relations for the own fields of the bunch. For the case $R_{\|}>R_{\perp}$, we can obtain

$$
E_{r}=\frac{3 e N r}{2\left(R_{\perp}^{2}-R_{\|}^{2}\right)^{3 / 2}} Q_{\perp}=-\frac{m a_{\perp}(t) r}{e},
$$

where

$$
\begin{gathered}
Q_{\perp}=\frac{R_{\|} \sqrt{R_{\|}^{2}-R_{\perp}^{2}}}{R_{\perp}^{2}}-\frac{1}{2} \ln \frac{R_{\|}+\sqrt{R_{\perp}^{2}-R_{\|}^{2}}}{R_{\|}-\sqrt{R_{\perp}^{2}-R_{\|}^{2}}}, \\
E_{z}=\frac{3 e N \zeta}{2\left(R_{\|}^{2}-R_{\perp}^{2}\right)^{3 / 2}} Q_{\|}=-\frac{m a_{\|}(t) \zeta}{e},
\end{gathered}
$$

where

$$
Q_{\|}=\ln \frac{R_{\|}+\sqrt{R_{\|}^{2}-R_{\perp}^{2}}}{R_{\|}-\sqrt{R_{\|}^{2}-R_{\perp}^{2}}}-2 \frac{\sqrt{R_{\|}^{2}-R_{\perp}^{2}}}{R_{\|}} .
$$

Time-dependent functions $a_{\perp}(t)$ and $a_{\|}(t)$ take part in the particle motion equations and in the equations for the bunch semiaxes $R_{\perp}, R_{\|}$[see (20)]. So Eqs. (20), (25), and (26) are the equations for the bunch semiaxes if the requirement $z_{0} \equiv$ const is satisfied $\left(z_{0}\right.$ is the longitudinal coordinate of the bunch mass center). Further, let us suppose there are both the external field and the own bunch field in the region considered, and the following condition is satisfied: $\operatorname{div} \overrightarrow{E^{\mathrm{ext}}}=0$. Let us consider the nonrotating bunch that moves along the axis $z$ in the external field. The field is supposed to be weakly nonuniform, the bunch dimensions being less than the characteristic length of the nonuniformity. The external field may be expanded in a series with respect to the radial distance between the particle position and the bunch center $\left[z=z_{0}(t), r=0\right]$. So the nonlinear summand of the series may be neglected. In the paraxial approximation the external field potential may be performed as $\Phi^{\mathrm{ext}} \cong \Phi_{0}(z)-\frac{r^{2}}{4} \Phi_{0}^{\prime \prime}(z)$. Let us use paraxial approximation for the external field force too. The external force in the same approximation is $\left.\left(z-z_{0}\right) \frac{\partial E_{z}}{\partial z}\right|_{z=z_{0}}=$ $\zeta \Phi_{0}^{\prime \prime}\left(z_{0}\right)$. This means that in Eq. (20) function $a_{\|}(t)$ must be replaced by $a_{\|}(t)+\frac{e}{m} \Phi_{0}^{\prime \prime}\left[z_{0}(t)\right]$, and function $a_{\perp}(t)$ respectively by $a_{\perp}(t)-\frac{e}{2 m} \Phi_{0}^{\prime \prime}\left[z_{0}(t)\right]$. If there is external field growth, transverse direction focusing appears, and on the contrary, the phase variation increases, e.g. the longitudinal defocusing is observed. If we will consider 
the motion of the bunch along the stationary magnetic field lines, we must replace the function $a_{\perp}(t)$ by the expression $a_{\perp}(t)+\left(\frac{e B}{2 m c}\right)^{2}$ in Eq. (20). Here $B$ is a value of the external stationary field. The replacement would be the same if the magnetic field is changing slowly and the dimensions of the bunch are quite small. In this case time dependence would look as $B(t)=B\left[z_{0}(t)\right]$, so the equation for $R_{\|}$is not changed and the longitudinal magnetic field affects the longitudinal bunch size implicitly by means of the transverse bunch size change.

\section{ZERO LONGITUDINAL EMITTANCE MODEL}

Formula (22) does not allow one to describe the bunch with zero longitudinal emittance, because $\varepsilon_{\|}$is placed in the denominator. So in this section another (rather simple) method of distribution function construction will be considered, resulting in uniform charge density of an ellipsoidal bunch. In the case of $\varepsilon_{\|}=0$, Eq. (19) is followed by the conclusion that invariant $I_{2}$ is a linear invariant in the second power. If $\ddot{\zeta}-a_{\|} \zeta=0$, then expression $I=$ $S(t) \dot{\zeta}-S(t) \zeta \equiv$ const, when function $S$ satisfies the next equation: $\quad S \ddot{(t} t)-a_{\perp}(t) S=0$. This equation has two linearly independent solutions. Let us sign them as $R_{\|}$ and $S_{1}$. Let us consider their Wronskian (time independent) equal to $w: S_{1}(t) \dot{R}_{\|}-S(t) R_{\|}(t) \equiv w$. Let us denote $I_{2}^{(1)}=$ $S_{1} \dot{\zeta}-\dot{S}_{1} \zeta, I_{2}^{(2)}=R_{\|} \dot{\zeta}-\dot{R}_{\|} \zeta$. The distribution function will be represented in the form of two $\delta$-functions product:

$$
\Psi\left(I_{1}, I_{2}^{(1)}, I_{2}^{(2)}\right)=\frac{3 N}{2 \pi} \delta\left[I_{1}+\left(I_{2}^{(1)}\right)^{2}-I_{0}\right] \delta\left(I_{2}^{(2)}\right),
$$

where $I_{1}$ is determined in (19). After integration with respect to the $\dot{\zeta}$ the single $\delta$-function stays with the argument

$$
\begin{aligned}
I_{1}+\zeta^{2} w^{2} / R_{\|}^{2}-I_{0}= & \left(\dot{r} R_{\perp}-r \dot{R_{\perp}}\right)^{2}+\mu^{2}\left(R_{\perp} / r\right)^{2} \\
& +\varepsilon_{\perp}^{2}\left(r / R_{\perp}\right)^{2}+\zeta^{2} w^{2} / R_{\|}^{2}-I_{0} .
\end{aligned}
$$

If $I_{0}=\varepsilon_{\perp}^{2}=w^{2}$ after integration with respect to the $\dot{r}$ and with respect to the $\mu$, we obtain for the density

$$
n=\frac{3 N}{4 \pi R_{\perp}^{2} R_{\|}} \sigma\left(1-\frac{r^{2}}{R_{\perp}^{2}}-\frac{\zeta^{2}}{R_{\|}^{2}}\right) .
$$

Note, that the model constructed by the same method can describe the bunch without axial symmetry. In paper [9] the problem of emittance transformation in the transverse magnetic field was studied by means of such a model. Combined usage of linear and square invariants for $3 \mathrm{D}$ bunches was considered in [10] too.

\section{CONCLUSION}

In the paper the different variants of kinetic models are represented describing the charged particle bunch shaped as an arbitrary ellipsoid with uniform particle density. In these models the own bunch fields are linear and may be considered along with external linear fields. The equations for the bunch envelopes are obtained in the case of weakly nonuniform external field too.

[1] E. A. Perelstein and G. D. Shirkov, Tech. Phys. 48, 249 (1978).

[2] I. M. Kapchinsky and V. V. Vladimirsky, In Proceedings of the Conference on High Energy Accelerators and Instrumentation, CERN, 1959 (CERN, Geneva, 1959), p. 274.

[3] A.S. Chikhachev, Kinetic Theory of Quasistationary States of Charged Particle Beams (Fizmatlit, Moscow, 2001) (in Russian).

[4] I. M. Kapchinsky, Theory of Linear Resonance Accelerators: Particle Dynamics (Energoatomizdat, Moscow, 1982) (in Russian).

[5] V. Danilov, S. Cousineau, S. Henderson, and J. Holmes, Phys. Rev. ST Accel. Beams 6, 094202 (2003).

[6] A. S. Chikhachev, Tech. Phys. 51, 496 (1981); 53, 1513 (1983).

[7] A.S. Chikhachev, Tech. Phys. 54, 1694 (1984).

[8] W. R. Smythe, Static and Dynamic Electricity (McGraw-Hill, New York, 1968), 3rd ed.

[9] H. Ye. Barminova and A.S. Chikhachev, Radiophys. Quantum Electron. 34, 818 (1991).

[10] Yu. A. Budanov and V.I. Shvetsov, in Proceedings X All-Russian Charged Particle Accelerator Conference, Dubna, 1986, Part 1, Dubna, 1987. 